\title{
A Clustering Based Method for Edge Detection in Hyperspectral Images
}

\author{
V.C. Dinh ${ }^{1,2}$, Raimund Leitner ${ }^{2}$, Pavel Paclik ${ }^{3}$, and Robert P.W. Duin ${ }^{1}$ \\ 1 ICT Group, Delft University of Technology, Delft, The Netherlands \\ 2 Carinthian Tech Research AG, Villach, Austria \\ 3 PR Sys Design, Delft, The Netherlands
}

\begin{abstract}
Edge detection in hyperspectral images is an intrinsically difficult problem as the gray value intensity images related to single spectral bands may show different edges. The few existing approaches are either based on a straight forward combining of these individual edge images, or on finding the outliers in a region segmentation. As an alternative, we propose a clustering of all image pixels in a feature space constructed by the spatial gradients in the spectral bands. An initial comparative study shows the differences and properties of these approaches and makes clear that the proposal has interesting properties that should be studied further.
\end{abstract}

\section{Introduction}

Edge detection plays an important role in image processing and analyzing systems. Success in detecting edges may have a great impact on the result of subsequent image processing, e.g. region segmentation, object detection, and may be used in a wide range of applications, from image and video processing to multi/hyper-spectral image analysis. For hyperspectral images, in which channels may provide different or even conflicting information, edge detection becomes more important and essential.

Edge detection in gray-scale images has been thoroughly studied and is well established. But for color images, especially multi-channel images like hyperspectral images, this topic is much less developed since even defining edges for those images is already a challenge 11. Two main approaches to detect edges in multi-channel images based on monochromatic [23] and vector techniques 4 456] have been published. The first detects edges in each individual band, and then combines the results over all bands. The latter, which has been proposed recently, treats each pixel in a hyperspectral image as a vector in the spectral domain, then performs edge detection in this domain. This approach is more efficient than the first one since it does not suffer from the localization variability of edge detection result in the individual channel. Therefore, in the scope of this paper, we mainly focus on the vector based approach.

Zenzo [4] proposed a method to extend the edge detection for gray-scale images to multi-channel images. The main idea is to find the direction for a point $\mathrm{x}$ for which its vector in the spectral domain has the maximum rate of change. 
Therefore, the largest eigenvalue of the covariance matrix of the set of partial derivatives at a pixel is selected as its edge magnitude. A thresholding method can be applied to reveal the edges. However, this method is sensitive to small texture variations as gradient-based operators are sensitive even to small changes. Moreover, determining the scale for each channel is another problem since the derivatives taken for different channels are often scaled differently.

Inspired by the work of using morphological edge detectors for the edge detection in gray-scale images [7], Trahanias et al. [5] suggested vector-valued ranking operators to detect edges in color images. First, they divided the image into small windows. For each window, they ordered the vector-valued data of pixels belonging to this window in increasing order based on the R-ordering algorithm [8]. Then, the vector range (VR), which can be considered as the edge strength, of every pixel is calculated as the deviation of the vector outlier in the highest rank to the vector median in the window. Different from Trahanias et al.'s method, Evans et al. 6] defined the edge strength of a pixel as the maximum distance between any two pixels within the window. Therefore, it helps to localize edge locations more precisely. However, the disadvantage of this method is neighborhood pixels often have same edge strength values since the window's space to find the edge strength of the two pixels are highly overlapping. As a result, it may create multiple responds for a single edge and the method is sensitive to noise.

These three methods could also be classified as model based or non-statistical approaches as they are designed by assuming a model of edges. Typical model based method can be mentioned as Canny's method [9], in which edges are assumed to be step functions corrupted by additive Gaussian noise. This assumption is often wrong for natural images which have highly structured statistical properties 101112. For a hyperspectral dataset, the number of channels can be up to hundreds, while the number of pixels in each channel can be easily up to millions. Therefore, how to exploit statistical information in both spatial and spectral domains of hyperspectral images is a challenging issue. However, there have been not much works on hyperspectral edge detection cornering this issue until now. Initial work on statistical based approach for edge detection in color image can be mentioned as Huntsberger et al. 13. They considered each pixel as a point in the feature space. A clustering algorithm is applied for a fuzzy segmentation of the image and then outliers of the clusters are considered as edges. However, this method performs image segmentation rather than edge detection and often produces discontinuous edges.

This paper proposes as an alternative a clustering based method for edge detection in hyperspectral images that could overcome the problem of Huntsberger et al.'s method. It is well-known that the pixel intensity is good for measuring the similarity among pixels, and therefore it is good for the purpose of image segmentation. But it is not good for measuring the abrupt changes to find the edges. The pixel gradient value is much more appropriate for that. Therefore, in our approach, we first consider each pixel as a point in the spectral space composed of gradient values in all image bands, instead of intensity values. Then, a 
clustering algorithm is applied in the spectral space to classify edge and non-edge pixels in the image. Finally, a thresholding strategy similar to Canny's method is used to refine the results.

The rest of this paper is organized as follows: Section 2 presents the proposed method for edge detection in hyperspectral images. To demonstrate its effectiveness, experimental results and comparisons with other typical methods are given in Section 3. In Section 4, some concluding remarks are drawn.

\section{Clustering Based Edge Detection in Hyperspectral Images}

First, the spatial derivatives of each channel in a hyperspectral images are determined. From 141, it is well-known that the use of fixed convolution masks of $3 \times 3$ size pixels is not suitable for the complex problem of determining discontinuities in image functions. Therefore, we use the 2-D Gaussian blur convolution to determine the partial derivatives. The advantage of using the Gaussian function is that we could reduce the effect of noise, which commonly occurs in hyperspectral images.

After the spatial derivatives of each channel are determined, gradient magnitudes of the pixels are calculated using the hypotenuse functions. Then each pixel can be considered as a point in the spectral space, which includes gradient magnitudes over all channels of the hyperspectral images. The problem of finding edges in the hyperspectral images could be considered as the same problem as classifying points in a spectral space into two classes: edge and non-edge points. We then use a clustering method based on the k-means algorithm for this classification purpose.

One important factor in designing the k-means algorithm is determining the number of clusters $N$. Formally, $N$ should be two as we distinguish edges and non-edges. However, in fact, the number of non-edge pixels often dominates the pixel population (from $75 \%$ to $95 \%$ ). Therefore, setting the number of clusters to two often results in losing edges since points in spectral space tend to belong to non-edge clusters rather than edge clusters. In practise, $N$ should be set to be larger than two. In this case, the cluster with the highest population is considered as the non-edge cluster. The remaining $N-1$ clusters are merged together and considered as the edge cluster. In our experiments, the number of clusters $N$ is set in the range of $[4.0,8.0]$. Experiments show that the edge detection results do not change much when $N$ is in this range.

After applying the k-means algorithm to classify each point in spectral space into one of $N$ clusters, a combined classifier method proposed by Paclik et al. [15] is applied to remove noise as well as isolated edges. The main idea of this method is to combine the results of two separate classifiers in spectral domain and spatial domain. This combining process is repeated until achieving stable results. In the proposed method, the results of two classifiers are combined using the maximum combination rule.

A thresholding algorithm as in the Canny edge detection method 9] is then applied to refine results from the clustering step, e.g. to make the edges thinner. 
There are two different threshold values in the thresholding algorithm: a lower threshold and a higher threshold. Different from Canny's method, in which the threshold values are based on gradient intensity, the proposed threshold values are determined based on the confidence of a pixel belonging to the non-edge cluster. A pixel in the edge cluster is considered as a "true" edge pixel if its confidence to the non-edge cluster is smaller than the lower threshold. A pixel is also considered as an edge pixel if it satisfies two criteria: its confidence to the non-edge cluster is in a range between the two thresholds and it has a spatial connection with an already established edge pixel. The remaining pixels are considered as non-edge pixels. Confidence of a pixel belonging to a cluster used in this step is obtained from the clustering step.

The proposed algorithm is briefly described as followings:

Algorithm 1. Edge detection for hyperspectral images

Input: A hyperspectral image $I$, number of clusters $N$. Output: Detected edges of the image as an image map. Step 1:

- Smoothing the hyperspectral image using Gaussian blur convolution.

- Calculating pixel gradient values in each image channel.

- Forming pixel as a point composed of gradient values over all bands in a feature space. The number of dimensions in the feature space is equal to the number of bands in the hyperspectral images.

Step 2: Applying the k-means algorithm to classify points into $N$ clusters. Step 3: Refining the clustering result using the combined classifier method. Step 4: Selecting the highest population cluster as non-edge cluster, merge other clusters as an edge cluster.

Step 5: Mapping the thresholding algorithm to refine results from Step 4.

\section{Experimental Results}

\subsection{Datasets}

Two typical hyperspectral datasets from [16] have been used for evaluating the performance of the proposed method. The first is a hyperspectral image of Washington DC Mall. The second is the "Flightline C1 (FLC1)" dataset taken from the southern part of Tippecanoe County, Indiana by an airborne scanner [16]. The properties of the two datasets are shown in the Table 1.

Since the spatial resolution of the two datasets is too large for handling it directly, we split the first dataset into 20 small parts of size $128^{*} 153$ and carry out experiments with each of the small ones. Similarly, we split the second dataset into 3 small parts of size $316^{*} 220$.

These two datasets are significantly diverse to evaluate the edge detector's performance. The first contains various types of regions, i.e. roofs, roads, paths, 
Table 1. Properties of datasets used in experiments

\begin{tabular}{c|c|c|c}
\hline \hline Dataset & No. channels & Spatial Resolution & Response $(\mu m)$ \\
\hline DC Mall & 191 & $1280^{*} 307$ & $0.4-2.4$ \\
FLC1 & 12 & $949^{*} 220$ & $0.4-1.0$ \\
\hline
\end{tabular}

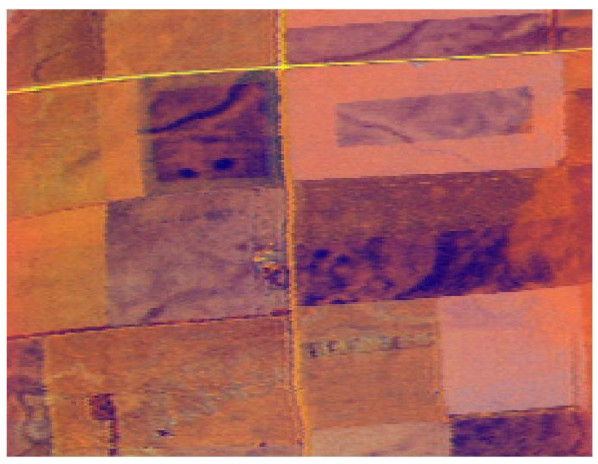

(a)

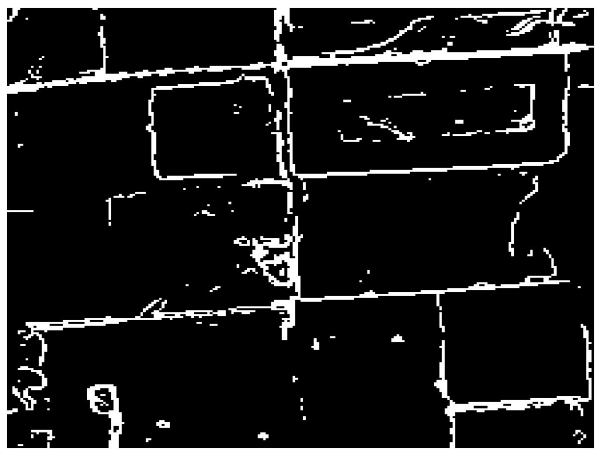

(c)

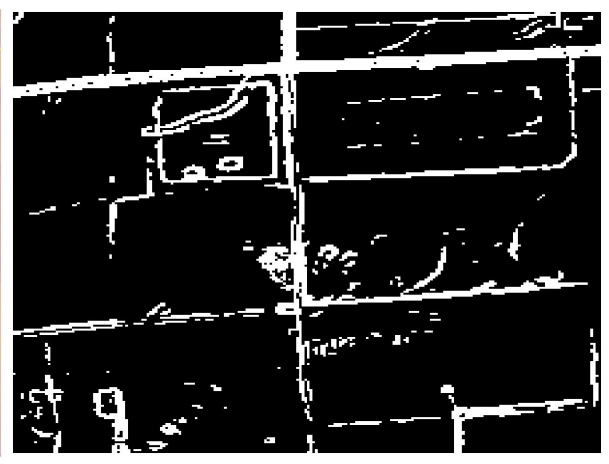

(b)

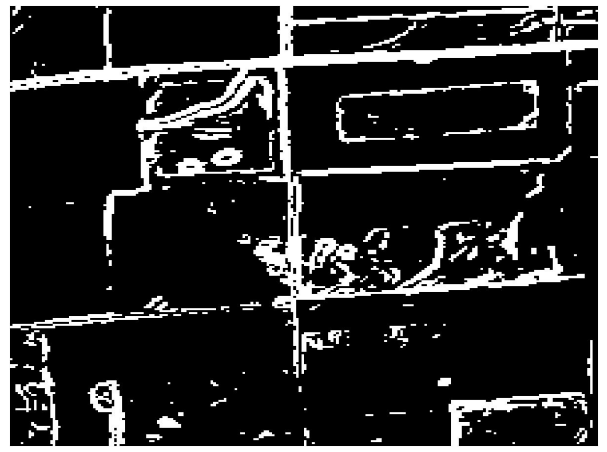

(d)

Fig. 1. Edge detection results on FLC1 dataset: dataset represented using PCA (a); edge detection results from Zenzo's method (b), Huntsberger's method (c), and the proposed method $(\mathrm{d})$

trees, grass, water, and shadows and has a large number of channels, while the second contains much simpler scene and has a moderate number of channels.

To provide the intuitive representations of these datasets, PCA is used. For each dataset, the first three principle components extracted by PCA are used to compose a RGB image. The first, second, and the third most important component corresponds to the Red, Green, Blue channels, respectively. Color representation of the two dataset are shown in Fig. 11(a) and Fig. 2(a). 


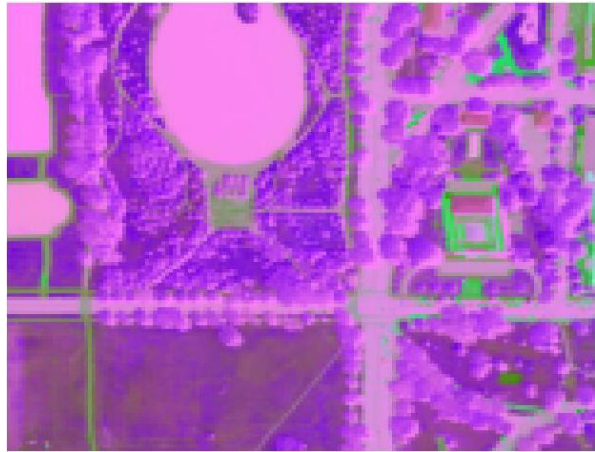

(a)

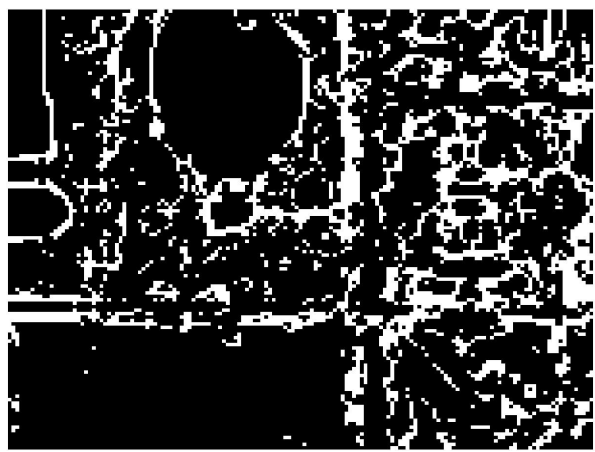

(c)

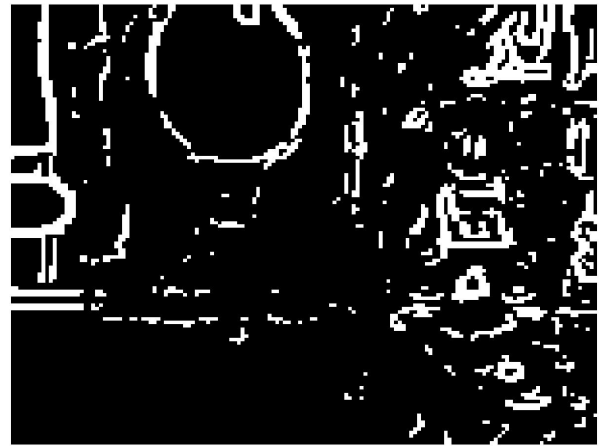

(b)

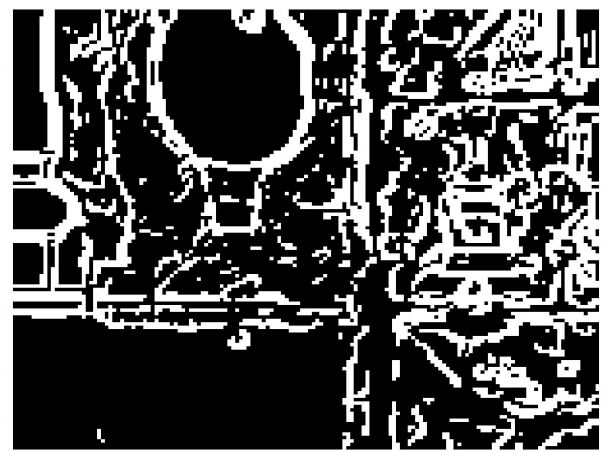

(d)

Fig. 2. Edge detection results on DC Mall dataset: dataset represented using PCA (a); edge detection results from Zenzo's method (b), Huntsberger's method (c), and the proposed method (d)

\subsection{Results}

In order to evaluate the effectiveness of the proposed method, we have compared it with two typical edge detection methods: Zenzo's method 4, a gradient based method, and a method presented by Huntsberger [13], an intensity clustering based method.

To provide a fair comparison, we carry out experiments with different parameter values for each edge detection method in both datasets and select the most suitable one. Moreover, we fix the parameter values for each method and use them for all datasets. For Zenzo's method, the threshold $t$ was set to the value that satisfies the number of pixels of which gradient strengths larger that $t$ is equal to $25 \%$ of the total number of pixels in spatial domain of the hyperspectral image. For Huntsberger 's method, the number of clusters is set to 5 , and the confident value of pixels with respect to the background cluster is set to 0.55 . For the proposed method, we apply Gaussian blur convolution for every channel 
of hyperspectral images with the standard deviation equal to 1 . The number of clusters is set to 6 .

Experimental results on the two datasets are shown in Fig. 1 and Fig. 2(b)2(d). It can be seen from the figures that Huntsberger's method performs worst: losing edges and creating discontinuous edges. Therefore, we will focus on the performance between Zenzo's method and the proposed method.

For the first dataset, which contains simple images, the two methods produce similar results. But for the second dataset, which contains a complex image, it is clear that the proposed method can preserve more local edges than Zenzo's method. It is because the proposed method makes use of statistical information in spectral space defined by multivariate gradients. Therefore, it works well even with noisy or low contrast images.

\section{Conclusions}

A clustering based method for edge detection in hyperspectral images is proposed. The proposed method enables the use of multivariate statistical information in multi-dimensional space. Based on pixel gradient values, it also provides a better representation of edges comparing to those based on intensity values, e.g. Huntsberger's method [13. As the results, the method reduces the effect of noise and preserves more edge information in the images. Experimental results, though still at preliminary work, show that the proposed method could be used effectively for edge detection in hyperspectral images. More thorough investigation in stabilizing the clustering methods and how to determine the number of clusters $N$ must be further invested to improve the results.

\section{Acknowledgements}

The authors would like to thank Sergey Verzakov, Yan Li, and Marco Loog for their useful discussions. This research is supported by the CTR, Carinthian Tech Research AG, Austria, within the COMET funding programme.

\section{References}

1. Koschan, A., Abidi, M.: Detection and classification of edges in color images. Signal Processing Magazine, Special Issue on Color Image Processing 22, 67-73 (2005)

2. Robinson, G.: Color edge detection. Optical Engineering, 479-484 (1977)

3. Hedley, M., Yan, H.: Segmentation of color images using spatial and color space information. Journal of Electronic Imaging 1, 374-380 (1992)

4. Di Zenzo, S.: A note on the gradient of a multi-image. Computer Vision, Graphics, and Image Processing, 116-125 (1986)

5. Trahanias, P., Venetsanopoulos, A.: Color edge detection using vector statistics. IEEE Transactions on Image Processing 2, 259-264 (1993)

6. Evans, A., Liu, X.: A morphological gradient approach to color edge detection. IEEE Transactions on Image Processing 15(6), 1454-1463 (2006) 
7. Haralick, R., Sternberg, S., Zhuang, X.: Image analysis using mathematical morphology. IEEE Transactions on Pattern Analysis and Machine Intelligence 9(4), 532-550 (1987)

8. Barnett, V.: The ordering of multivariate data. J. Royal Statist., 318-343 (1976)

9. Canny, J.: A computational approach to edge detection. IEEE Transactions on Pattern Analysis and Machine Intelligence, 679-698 (1986)

10. Field, D.: Relations between the statistics and natural images and the responses properties of cortical cells. Journal of Optical Society of America A(4), 2379-2394 (1987)

11. Zhu, S.C., Mumford, D.: Prior learning and gibbs reaction-diffusion. IEEE Transactions on Pattern Analysis and Machine Intelligence 19(11), 1236-1250 (1997)

12. Konishi, S., Yuille, A.L., Coughlan, J.M., Zhu, S.C.: Statistical edge detection: Learning and evaluating edge cues. IEEE Transactions on Pattern Analysis and Machine Intelligence 25(1), 57-74 (2003)

13. Huntsberger, T., Descalzi, M.: Color edge detection. Pattern Recognition Letter, 205-209 (1985)

14. Marr, D., Hildreth, E.: Theory of edge detection. Proceedings of Royal Society of London, 187-217 (1980)

15. Paclik, P., Duin, R.P.W., van Kempen, G.M.P., Kohlus, R.: Segmentation of multispectral images using the combined classifier approach. Journal of Image and Vision Computing 21, 473-482 (2005)

16. Landgrebe, D.: Signal theory methods in multispectral remote sensing. John Wiley and Sons, Chichester (2003) 